\title{
STUDIES IN FABER POLYNOMIALS. I
}

\author{
BY \\ JOSEPH L. ULLMAN
}

1. Introduction. The research on the following problem was suggested by Dr. G. Szegö, to whom much is owed, both for general encouragement and for many specific suggestions. In 1903, in his thesis, G. Faber $\left({ }^{1}\right)$ [1] introduced polynomials, which have since proved useful in Analysis, and have become known as Faber polynomials. The results of the following research concern the asymptotic distribution of the zeros of Faber polynomials.

Definition 1.1. Let $f(z)$ be a power series of the type

$$
z+a_{0}+\frac{a_{1}}{z}+\cdots
$$

where the coefficients satisfy the condition that $\lim \sup \left|a_{n}\right|^{1 / n}<\infty$. For each $n \geqq 1$, form

$$
[f(z)]^{n}=F_{n}(z)+R_{n}(z),
$$

where $F_{n}(z)$ is a polynomial of degree $n$ and $R_{n}(z)$ contains only negative powers of $z$. The polynomials $F_{n}(z)$ are the Faber polynomials associated with $f(z)$.

This is equivalent to Faber's original definition, except that he specified that $f(z)$ be the Taylor's series expansion about $z=\infty$ of the normalized exterior mapping function of a simple Jordan curve $C$ in the $z$-plane. He referred to the polynomials as being associated with the curve $C$. In his thesis, he proved that an arbitrary analytic function $G(z)$, regular in the interior of $C$, could be expanded as a series of the Faber polynomials associated with $C$,

$$
G(z)=\sum_{0}^{\infty} a_{n} F_{n}(z),
$$

such that the series converges to $G(z)$ uniformly in any closed subset of the interior of $C$. In proving this result, Faber derived and used the generating function for the polynomials. It is derived here by a method adapted to later applications.

Consider a series of type (1.1), with $\lim \sup \left|a_{n}\right|^{1 / n}<\infty$. Because of the restriction on the coefficients, the series converges uniformly in a neighborhood of $z=\infty$. Indeed, a positive number $\gamma$ can be chosen, so that in the closed exterior of the circle $\Gamma,|z|=\gamma$, the series defines a function that is called $w=f(z)$ without ambiguity, which is analytic except for a simple pole

Received by the editors February 25, 1959.

(1) Numbers in brackets refer to the bibliography at the end of the paper. 
at $z=\infty$, and performs a 1-1 mapping of the exterior of $\Gamma$ onto the exterior of a simple analytic curve $\Gamma^{\prime}$. These properties are preserved if $\gamma$ is replaced by a larger number, so we can assume that $\gamma>|\zeta|$, where $\zeta$ is an arbitrary complex number. Consider the integral

$$
I(\zeta)=\frac{1}{2 \pi i} \int_{\Gamma^{+}} \frac{[f(z)]^{n}}{z-\zeta} d z .
$$

Substitute (1.2) to obtain

$$
I(\zeta)=\frac{1}{2 \pi i} \int_{\Gamma^{+}} \frac{F_{n}(z)}{z-\zeta} d z+\frac{1}{2 \pi i} \int_{\Gamma^{+}} \frac{R_{n}(z)}{z-\zeta} d z .
$$

Since the series $f(z)$ represents an analytic function for $z$ in the closed exterior of $\Gamma$, the same is true of $[f(z)]^{n}$, and therefore $R_{n}(z)$. Since $R_{n}(z)$ contains only negative powers of $z, R_{n}(z)=O(1 /|z|)$. In the second integral on the right, the value is not changed by replacing $\Gamma$ by a circle of radius $\rho$, where $\rho>\gamma$. Since $R_{n}(z)=O(1 /|z|)$, the integral tends to zero as $\rho \rightarrow \infty$, and must therefore equal zero. Since $\zeta$ is in $\Gamma,(1.5)$ becomes

$$
I(\zeta)=F_{n}(\zeta)=\frac{1}{2 \pi i} \int_{\Gamma^{+}} \frac{[f(z)]^{n}}{z-\zeta} d z .
$$

Next, make the change of variable $z=g(w)$ in (1.6) where $g(w)$ is the inverse function of $f(z)$, obtaining

$$
F_{n}(\zeta)=\frac{1}{2 \pi i} \int_{\Gamma_{1}^{+}} \frac{w^{n} g^{\prime}(w)}{g(w)-\zeta} d w .
$$

The function $g^{\prime}(w) /(g(w)-\zeta)$ has a simple zero at $w=\infty$, and therefore has an expansion of the type

$$
\frac{g^{\prime}(w)}{g(w)-\zeta}=\sum_{0}^{\infty} \frac{b_{n}(\zeta)}{w^{n+1}}
$$

Equation (1.7) is the Cauchy integral formula for the coefficient of $1 / w^{n+1}$ in the right hand side of $(1.8)$, hence $b_{n}(\zeta)=F_{n}(\zeta)$ and

$$
\frac{g^{\prime}(w)}{g(w)-\zeta}=\sum_{0}^{\infty} \frac{F_{n}(\zeta)}{w^{n+1}} .
$$

This is the generating function of the Faber polynomials. The domain of convergence of the right hand side of (1.9) depends on $\zeta$. The exact relationship between the radius of convergence of the series in (1.9) and the value of $\zeta$ is derived in $\S 4$.

2. The zeros in a special case. Let $F(z)$ be the branch of $\left(z^{2}-1\right)^{1 / 2}$ which is holomorphic in the $z$-plane cut along the real axis from $z=-1$ to $z=1$, and 
is positive for $z$ real and greater than one. Let $f(z)$ also designate the Taylor's series expansion about $z=\infty$ of $\mathcal{F}(z)$. We study the Faber polynomials associated with $f(z)$ in this section. The following results on the zeros of partial sums of Taylor's series are used. Let $h(z)=\sum_{0}^{\infty} a_{k} z^{k}$, lim sup $\left|a_{k}\right|^{1 / k}=1$. Let $h_{n}(z)=\sum_{0}^{n} a_{k} z^{k}$ have the zeros $z_{n i}(i=1, \cdots, n)$ which we designate by $\left(z_{n i}\right)$. Let $\left\{\left(z_{n i}\right)\right\}$ denote the sequence of sets of points $\left(z_{1, i}\right),\left(z_{2, i}\right), \cdots$. R. Jentzsch [3] proved that every point of the circle $|z|=1$ is a limit point of the elements of $\left\{\left(z_{n i}\right)\right\}$. Later G. Szegö [4] proved a generalization which we refer to as the Szegö-Jentzsch theorem. Consider only zeros of sections $h_{n_{\nu}}(z)$ for which $\lim \left|a_{n_{\nu}}\right| 1 / n_{\nu}=1$, indicating them by $\left\{\left(z_{n_{\nu}, i}\right)\right\}$. These points are distributed with the following properties. Let $r(n)$ be a function which is $o(n)$. For any $\epsilon>0$, the points $\left(z_{n_{y}, i}\right)$ satisfy ||$z_{n_{y}, i}|-1|<\epsilon$, except for at most $r(n)$ values of $i$. Let $\arg z_{n_{v}, i}=\theta_{n_{\nu}, i}$, let $\alpha$ and $\beta$ be numbers satisfying $0 \leqq \alpha<\beta<2 \pi$ and let $\lambda_{n_{\nu}}(\alpha, \beta)$ be the number of $\theta_{n_{\nu}, i}$ satisfying $\alpha \leqq \theta_{n_{\nu}, i} \leqq \beta$, then

$$
\lambda_{n_{\nu}}(\alpha, \beta)=n_{\nu}\left(\frac{\beta-\alpha}{2 \pi}\right)+o\left(n_{\nu}\right) .
$$

Points $\left\{\left(z_{n_{y}, i}\right)\right\}$ satisfying these two properties are said to be uniformly distributed about the circle $|z|=1$. A greater degree of generality is required to discuss the zeros of the Faber polynomials of $f(z)$.

Let $\Gamma$ be a curve consisting of finitely many analytic arcs $\Gamma_{1}, \cdots, \Gamma_{k}$, touching only at their endpoints, and forming the boundary of a simply connected domain $D$ which contains the point $z=\infty$. Let $w=F(z)$ be the holomorphic function which maps $D$ conformally onto the exterior of the circle $\Sigma,|w|=1$, in a one-to-one manner, taking $z=\infty$ into $w=\infty$ and satisfying the condition

$$
\lim _{z \rightarrow \infty} \frac{F(z)}{z}=k,
$$

where $k$ is a positive constant. Let $\Gamma^{\prime}$ be the set of points of $\Gamma$ at a distance greater than $\delta$ from the end points of the $\operatorname{arcs} \Gamma_{i}$. Let $D^{*}$ be the domain consisting of $D$ and every other point in the $z$-plane whose distance from $\Gamma$ is less than $\gamma$. For any $\delta, \gamma$ can be chosen so that $D^{*}$ is simply covered, and so that $F(z)$ can be uniquely continued into $D^{*}$ by the reflection principle and remain one-to-one. Call this holomorphic function $F^{*}(z)$.

A sequence of sets of points $\left\{\left(z_{n_{y}, i}\right)\right\},\left(n_{1}<n_{2}<\cdots ; i=1, \cdots, n\right)$ is said to be conformally distributed about the curve $\Gamma$ if it has the following properties. Let $s(n)$ be a function which is $o(n)$. For any $\epsilon>0$, the points $\left(z_{n_{p}, i}\right)$ must be within a distance $\epsilon$ of $\Gamma$, except for at most $s(n)$ values of $i$. Let $(a, b)^{\wedge}$ be a closed subarc of $\Gamma$ consisting of interior points of one of the $\operatorname{arcs} \Gamma_{i}$, and whose image on $\Sigma$ is $\left(e^{i \alpha}, e^{i \beta}\right)^{\wedge}$. Choose $\delta$ so that $a, b$ is contained 
in $\Gamma^{\prime}$ and then choose $\gamma$ so that $F^{*}(z)$ will be one-to-one in $D^{*}$. Let $\mu_{n_{y}}(a, b)$ be the number of $\left(z_{n_{v}, i}\right)$ for which $F^{*}(z)$ is defined and satisfies $\alpha \leqq \arg F^{*}\left(z_{n_{v}, i}\right)$ $\leqq \beta$. Then

$$
\mu_{n_{\nu}}(a, b)=n_{\nu}\left(\frac{\beta-\alpha}{2 \pi}\right)+o\left(n_{\nu}\right) .
$$

The Faber polynomials of $f(z)$ of even degree are given by

$$
F_{2 k}(z)=[f(z)]^{2 k}=\left[\left(z^{2}-1\right)^{1 / 2}\right]^{2 k}=\left(z^{2}-1\right)^{k} .
$$

Thus the polynomial of degree $2 k$ has a zero of multiplicity $k$ at $z=1$ and of multiplicity $k$ at $z=-1$. What we now prove is that the zeros of the polynomials of odd degree are all interior to the lemniscate $\Gamma,\left|z^{2}-1\right|=1$, except that each has a simple zero at $z=0$, and that the zeros are conformally distributed on $\Gamma$. We use the generating function (1.9). The inverse of $w=\mathcal{F}(z)$ is $z=\mathcal{G}(w)=\left(w^{2}+1\right)^{1 / 2}$, where $\mathcal{G}(w)$ designates the branch of $z=\left(w^{2}+1\right)^{1 / 2}$ which is holomorphic in the $w$-plane cut from $i$ to $-i$, and is positive for positive values of $w$. Hence

$$
\sum_{0}^{\infty} \frac{F_{n}(\zeta)}{w^{n+1}}=\frac{1}{\left(1+w^{-2}\right)^{1 / 2}} \cdot \frac{1}{w\left(1+w^{-2}\right)^{1 / 2}-\zeta} .
$$

The generating function of the polynomials of odd degree is obtained by replacing $w$ by $(-w)$ in $(2.4)$ and adding the function obtained to (2.4) as follows,

$$
\begin{gathered}
\sum_{0}^{\infty} \frac{F_{n}(\zeta)}{w^{n+1}}+\sum_{0}^{\infty} \frac{F_{n}(\zeta)}{(-w)^{n+1}} \\
=\frac{1}{\left(1+w^{-2}\right)^{1 / 2}} \cdot\left[\frac{1}{w\left(1+w^{-2}\right)-\zeta}+\frac{1}{-w\left(1+w^{-2}\right)^{1 / 2}-\zeta}\right] \\
\quad \sum_{0}^{\infty} \frac{F_{2 k+1}(\zeta)}{w^{2 k+2}}=\frac{\zeta}{\left(1+w^{-2}\right)^{1 / 2}} \cdot \frac{1}{w^{2}+1-\zeta^{2}} .
\end{gathered}
$$

Let $h\left(w^{2}\right)$ be the Taylor's expansion about $w=\infty$ of $\left(1+w^{-2}\right)^{-1 / 2}$, thus

$$
\begin{aligned}
h\left(w^{2}\right) & =\sum_{0}^{\infty} u_{k} w^{-2 k}, \\
u_{k} & =(-1)^{k} \frac{1 \cdot 3 \cdots 2 k-1}{2 \cdot 4 \cdots 2 k} .
\end{aligned}
$$

Let $h_{n}\left(w^{2}\right)=\sum_{0}^{n} u_{k} w^{-2 k}$. We expand the right side of (2.6) in powers of $w^{-2}$, use the Cauchy rule for product of power series, and obtain 


$$
\begin{aligned}
\frac{\zeta}{\left(1+w^{-2}\right)^{1 / 2}} \cdot \frac{1}{w^{2}-\left(\zeta^{2}-1\right)} & =\zeta \sum_{0}^{\infty} \frac{u_{k}}{w^{2 k}} \cdot \sum_{0}^{\infty} \frac{\left(\zeta^{2}-1\right)^{k}}{w^{2 k+2}} \\
& =\zeta \sum_{k=0}^{\infty} \frac{\sum_{j+l=k} u_{j}\left(\zeta^{2}-1\right)^{l}}{w^{2 k+2}} \\
& =\sum_{k=0}^{\infty} \frac{\zeta\left(\zeta^{2}-1\right)^{k} h_{k}\left(\zeta^{2}-1\right)}{w^{2 k+2}}
\end{aligned}
$$

Comparing coefficients of powers of $w$ in (2.6) and (2.8), we obtain the identity

$$
F_{2 k+1}(\zeta)=\zeta\left(\zeta^{2}-1\right)^{k} h_{k}\left(\zeta^{2}-1\right)
$$

The coefficients of the polynomial

$$
t^{k} h_{k}(t)=\sum_{j=0}^{k} u_{j} t^{k-j}
$$

decrease in magnitude, hence by a theorem of Kakeya-Eneström the $k$ zeros $t_{k 1}, \cdots, t_{k k}$, or $\left(t_{k i}\right)$, all have modulus less than one. The roots of $F_{2 k+1}(\zeta)$ are $\zeta_{2 k+1,2 k+1}=0$ and the $2 k$ solutions of $\zeta^{2}-1=t_{k i}(i=1, \cdots, k)$. Let the two solutions corresponding to $t_{k i}$ be indicated by $\zeta_{2 k+1,2 i-1}$ and $\zeta_{2 k+1,2 i}$. Hence $\left|\zeta_{2 k+1,2 i-1}^{2}-1\right|=\left|\zeta_{2 k+1,2 i}^{2}-1\right|=\left|t_{k i}\right|<1, \quad(i=1, \cdots, k)$, and the zeros of $F_{2 k+1}(\zeta)$ are inside the lemniscate $\Gamma$, except for the simple zero at $\zeta=0$. Since $\lim \left|u_{j}\right|^{1 / i}=1$, using the Szegö-Jentzsch theorem, we find that the $\left\{\left(t_{k i}\right)\right\}$ are uniformly distributed about the circle $|t|=1$. Hence, for any $\rho, 0<\rho<1$, $\left|t_{k i}\right|>\rho$, except for at most $r(k)$ values of $i$. Now the lemniscates $\Gamma_{\rho},\left|z^{2}-1\right|$ $=\rho$, converge uniformly to $\Gamma$ as $\rho$ tends to one. Thus, for any $\epsilon, \rho$ can be chosen so that those points of $\left(\zeta_{2 k+1, j}\right)$ which lie in the annulus between $\Gamma_{\rho}$ and $\Gamma$ are at a distance less than $\epsilon$ from $\Gamma$. Since there are at most $s(2 k+1)=2 r(k)$ points which do not lie in this annulus, the points $\left(\zeta_{2 k+1, i}\right)$ satisfy the first condition for conformal distribution about $\Gamma$. We now prove the second condition. The curve $\Gamma$ consists of two arcs, $\Gamma_{1}$ and $\Gamma_{2}$, one of which, say $\Gamma_{1}$, is in the half-plane $\operatorname{Re} z \geqq 0$. Let $(a, b)^{\wedge}$ be a closed sub-arc of interior points of $\Gamma_{1}$. Let $w=F(z)$ be the normalized exterior mapping function of $\Gamma$ and let $\left(e^{i \alpha}, e^{i \beta}\right)^{\wedge}$ be the image of $(a, b)^{\wedge},-\pi<\alpha<\beta<\pi$. Of the two solutions of $\zeta^{2}-1=t_{k i}$, say $\zeta_{2 k+1,2 i-1}$ is in the half-plane $\operatorname{Re} z>0$. Further, we note that the function $F(z)$ is the restriction of $F(z)$ to the exterior of $\Gamma$, which we denote by $D$. Also, $F^{*}(z)$ is the restriction of $\mathcal{F}(z)$ to any properly chosen domain $D^{*}$, since the function $F(z)$ is one-to-one in the $z$-plane cut from $z=-1$ to $z=1$. Now $\alpha \leqq \arg F\left(\zeta_{2 k+1, j}\right) \leqq \beta$ implies that $j$ is of the form $2 i-1$, and that the argument of a square root of $t_{k i}$ lies between $\alpha$ and $\beta$, or $2 \alpha \leqq \arg t_{k i}$ $\leqq 2 \beta$. Since $\left\{\left(t_{k i}\right)\right\}$ is uniformly distributed on $|t|=1$, there are by $(2.1)$, $k((2 \beta-2 \alpha) / 2 \pi)+o(k)$ solutions of this inequality. Furthermore, $F^{*}(z)$ is de- 
fined and coincides with $\mathcal{F}(z)$ for the $\zeta_{2 k+1, j}$ lying in $\operatorname{Re} z>0$ which correspond to these values of $t_{k i}$, except for at most $r(k)$ values. Hence

$$
\begin{aligned}
\mu_{2 k+1}(a, b) & =\lambda_{k}(\alpha, \beta)+r(k) \\
& =k \frac{2 \beta-2 \alpha}{2 \pi}+o(k)+r(k) \\
& =(2 k+1)\left(\frac{\beta-\alpha}{2 \pi}\right)+o(k),
\end{aligned}
$$

and condition (2.2) is satisfied.

3. The zeros in the general case. Attention is now restricted to $f(z)$, an arbitrary but fixed series of type

$$
z+a_{0}+\frac{a_{1}}{z}+\cdots,
$$

with $\lim \sup \left|a_{n}\right|^{1 / n}<\infty$.

Definition 3.1. Let $z_{n i}(i=1, \cdots, n)$ be the zeros of the Faber polynomial $F_{n}(z)$ associated with $f(z)$, and let $\Delta$ be the derived set of the $z_{n i}(n=1, \cdots$; $i=1, \cdots, n)$.

To describe the location of $\Delta$, the points of the $z$-plane are divided into classes, the method of classification depending on properties of $f(z)$ that are next to be discussed. Since the coefficients of (3.1) satisfy the condition $\lim$ sup $\left|a_{n}\right|^{1 / n}<\infty$, the series (3.1) defines an analytic element of an analytic function. Designate the analytic function by $w=f(z)$. In a neighborhood of $z=\infty$, the series (3.1) defines a portion of $w=f(z)$ which is single valued and univalent. Therefore, the inverse of this portion of $w=f(z)$ is uniquely determined in a neighborhood of $w=\infty$, and has the expansion

$$
w+b_{0}+\frac{b_{1}}{w}+\cdots,
$$

about the point $w=\infty$. The following notation will be used. For any $\eta \geqq 0$, let $\sigma_{\eta}$ be the circle $|w|=\eta, \Sigma_{\eta}$ the domain $|w|>\eta$, and $\bar{\Sigma}_{\eta}$ the region $|w| \geqq \eta$. If in (3.2), lim sup $\left|b_{n}\right|^{1 / n}=\rho$, then (3.2) converges uniformly in any closed subset of $\Sigma_{\rho}$, defining a holomorphic function. Thus, a holomorphic function is defined in a neighborhood of every point of $\Sigma_{\rho}$.

Definition 3.2. Let $z=g(w)$ be the holomorphic function defined by the series (3.2), in $\Sigma_{\rho}$.

Let $\zeta, \zeta^{\prime}, \ldots$ indicate complex constants in the $z$-plane, and $\omega, \omega^{\prime}, \ldots$ complex constants in the $w$-plane. Consider the equation

$$
g(w)-\zeta=0 .
$$

Say $\rho^{\prime}>\rho$. In $\Sigma \rho^{\prime},(3.3)$ has either no solution or finitely many solutions, hence 
the total number of solutions of (3.3) in $\Sigma_{\rho}$ is denumerable, and the solutions can be given an index $\omega_{i},(i=1,2, \cdots)$. Since the domain of definition of $z=g(w)$ is $\Sigma_{\rho},\left|\omega_{i}\right|>\rho$. A value $\omega_{i}$ is called a $\zeta$-point of $g(w)$. If $g^{\prime}\left(\omega_{i}\right) \neq 0$, it is called a simple $\zeta$-point, or a simple solution of (3.3).

DEFINITION 3.3. The values of $\zeta$ for which (3.3) has no solutions are called 0 -points, and belong to the set $C_{0}$. Next, consider a value for which (3.3) has solutions. If, among the $\zeta$-points of $g(w)$, there is one, say $\omega_{1}$, of greatest modulus, and if $\omega_{1}$ is a simple $\zeta$-point, then the value $\zeta$ is classified as a 1-point. The set of 1-points constitutes the set $C_{1}$ in the z-plane.

The further classification of points in the $z$-plane is carried out in $\$ 4$. Definition 3.3 is sufficient for the statement of Theorem 1, and for the proof of its first part.

TheOREM 1. The set $\Delta$ (a) lies in the complement of $C_{1}$, and (b) contains every boundary point of $C_{1}$.

Proof (a). It is first shown that $C_{1}$ contains a neighborhood of $z=\infty$, and that no point of $\Delta$ iies in this neighborhood.

Leммa 3.1. Given the function $z=g(w)$ of Definition 3.2 with domain of definition $\Sigma_{\rho}$ (i) there is $a \rho^{\prime}>\rho$ and $a P>0$, such that if $|\zeta| \geqq P, g(w)-\zeta=0$ has one solution in $\Sigma_{\rho^{\prime}}$, say $\omega$, and it is a simple solution. Furthermore, (ii) the value $\omega$ corresponding to $\zeta$ is given by a holomorphic function, $w=f(z, \infty, P)$, defined for $|z| \geqq P$.

A proof of (i) is given here, and the proof of (ii) is found in [2, pp. 135139]. Choose $\rho^{\prime}>\rho$. Let $\max |g(w)|=M$ for $w$ on $\sigma_{\rho^{\prime}}$. Choose $P>M$. If $\zeta$ is chosen so that $|\zeta| \geqq P$, then $|g(w)|<|\zeta|$ for $w$ on $\sigma_{\rho^{\prime}}$. The argument principle can then be used to show that $g(w)-\zeta=0$ has a simple solution in $\Sigma_{\rho^{\prime}}$, since $g(w)$ has a simple pole for $w=\infty$.

Now, if $|\zeta| \geqq P$, only one solution of $g(w)-\zeta=0$ is in $\Sigma_{\rho^{\prime}}$; hence, any other solution has a smaller modulus. Thus $\zeta$ is a 1 -point and the set $|z| \geqq P$ is contained in $C_{1}$. It is next shown that no point of $\Delta$ lies in the set $|z| \geqq P$.

Consider the integral

$$
I(\zeta)=\frac{1}{2 \pi i} \int_{e_{\rho^{\prime}}} \frac{w^{n} g^{\prime}(w)}{g(w)-\zeta} d w
$$

where $\zeta, \rho^{\prime}$ are constants introduced in Lemma 3.1. By the lemma, $\omega=f(\zeta, \infty, P)$ is the only solution of $g(w)-\zeta=0$ in $\Sigma_{\rho^{\prime}}$. Let $\rho^{\prime \prime}>|\omega|$, then

$$
I(\zeta)=\frac{1}{2 \pi i} \int_{\sigma_{\rho^{+}++\sigma_{\rho^{-}}}} \frac{w^{n} g^{\prime}(w)}{g(w)-\zeta} d w+\frac{1}{2 \pi i} \int_{\sigma_{\rho}^{+,}} \frac{w^{n} g^{\prime}(w)}{g(w)-\zeta} d w .
$$

The integrand has a simple pole at $w=\omega$ with residue $-(\omega)^{n}=-[f(\zeta, \infty, P)]^{\mathrm{n}}$. The second integral, by (1.6), is equal to $F_{n}(\zeta)$, hence 


$$
I(\zeta)=F_{n}(\zeta)-[f(\zeta, \infty, P)]^{n}
$$

It follows that

$$
\left|1-\frac{F_{n}(\zeta)}{f(\zeta, \infty, P)^{n}}\right| \leqq \frac{1}{2 \pi} \int_{\sigma_{\rho^{\prime}}}\left|\frac{w}{f(\zeta, \infty, P)}\right|^{n}\left|\frac{g^{\prime}(w) d w}{g(w)-\zeta}\right| .
$$

Now, $|g(w)-\zeta| \geqq|g(w)|-|\zeta| \geqq P-M$, for $w$ on $\sigma_{\rho^{\prime}}$. Also, $\min |f(z, \infty, P)|$ $>\rho^{\prime}+\delta,(\delta>0)$ for $|z| \geqq P$. In the contrary case, there would be a value $\zeta$, such that $|\zeta| \geqq P$, and $|\omega|=|f(\zeta, \infty, P)|=\rho^{\prime}$. Hence, it would follow that $g(\omega)=\zeta$ and thus $|g(\omega)| \geqq P$ for $w$ on $\sigma_{\rho^{\prime}}$, a contradiction.

Let $Q=\max \left|g^{\prime}(w)\right|$ for $w$ on $\sigma_{\rho^{\prime}}$. It then follows that

$$
\frac{1}{2 \pi} \int_{\sigma_{\rho^{\prime}}}\left|\frac{w}{f(\zeta, \infty, P)}\right|^{n}\left|\frac{g^{\prime}(w) d w}{g(w)-\zeta}\right| \leqq\left(\frac{\rho^{\prime}}{\rho^{\prime}+\delta}\right)^{n} \frac{Q_{\rho^{\prime}}}{(P-M)} .
$$

Hence, for $n>n_{0}$

$$
\left|1-\frac{F_{n}(\zeta)}{f(\zeta, \infty, P)}\right|<1
$$

The estimates used in (3.8) are independent of $\zeta$, if $|\zeta| \geqq P$, hence, (3.9) is true for $|\zeta| \geqq P$. Thus, $F_{n}(\zeta) \neq 0$ for $|\zeta| \geqq P$ and $n>n_{0}$, and, therefore, no point of $\Delta$ lies in the set $|z| \geqq P$.

It is next shown that if $\zeta$ is a finite complex number in $C_{1}$, then a neighborhood of $\zeta$ belongs to $C_{1}$, and that no point of $\Delta$ lies in this neighborhood.

Lemma 3.2. Given the function $z=g(w)$ of Definition 3.2, with domain of definition $\Sigma_{\rho}$, let $\zeta$ be a simple 1-point, and $\omega$ the $\zeta$-point of $g(w)$ of greatest modulus. (i) There is $a \rho^{\prime}>\rho$ and $a \delta>0$, such that if $\left|\zeta^{\prime}-\zeta\right| \leqq \delta, g(w)-\zeta^{\prime}=0$ has one solution $\omega$ in $\Sigma_{\rho^{\prime}}$ and it is a simple solution. Furthermore, (ii) the values of $\omega$ are given by a holomorphic function $w=f(z, \zeta, \delta)$, defined for $|z-\zeta| \leqq \delta$.

It is sufficient to give a sketch of (i), since (ii) is proved in [2, pp. 135139]. The number $\rho^{\prime}$ can be chosen so that $\rho<\rho^{\prime}<|w|$, and so that no other $\zeta$ points of $g(w)$ lie in $\bar{\Sigma}_{\rho^{\prime}}$. Say min $|g(w)-\zeta|=2 \delta$ for $w$ on $\sigma_{\rho^{\prime}}$, with $\delta>0$. Then, if $\left|\zeta^{\prime}-\zeta\right| \leqq \delta, g(w)-\zeta^{\prime}=0$ has one solution in $\Sigma_{\rho^{\prime}}$, and it is simple. This is a consequence of the argument principle.

Thus, if $\left|\zeta^{\prime}-\zeta\right| \leqq \delta, g(w)-\delta=0$ has one solution in $\Sigma_{\rho^{\prime}}$; hence, any other solution has smaller modulus. Furthermore, the solution is simple. Hence, $\zeta^{\prime}$ is a 1 -point, and the set $|z-\zeta| \leqq \delta$ belongs to $C_{1}$.

Since $\zeta^{\prime}$ is a simple 1-point, by Lemma 3.2, the $\zeta^{\prime}$-point of $g(w)$ of greatest modulus is given by $\omega^{\prime}=f\left(\zeta^{\prime}, \zeta^{\prime}, \delta^{\prime}\right), \delta^{\prime}$ depending on $\zeta^{\prime}$. On the other hand, since $\left|\zeta^{\prime}-\zeta\right| \leqq \delta, \zeta^{\prime}$ lies in the domain of definition of $f(z, \zeta, \delta)$, and hence $\omega^{\prime}=f\left(\zeta^{\prime}, \zeta, \delta\right)$.

Consider the integral 


$$
I(\zeta)=\frac{1}{2 \pi i} \int_{\sigma_{\rho^{\prime}}} \frac{w^{n} g^{\prime}(w)}{g(w)-\zeta} d w .
$$

In a similar fashion to the treatment of (3.5), this becomes

$$
I(\zeta)=F_{n}(\zeta)-[f(\zeta, \zeta, \delta)]^{n}
$$

If $\zeta^{\prime}$ is a constant satisfying $\left|\zeta^{\prime}-\zeta\right| \leqq \delta$, then

$$
\begin{aligned}
I\left(\zeta^{\prime}\right) & =F_{n}\left(\zeta^{\prime}\right)-\left[f\left(\zeta^{\prime}, \zeta^{\prime}, \delta^{\prime}\right)\right]^{n} \\
& \geqq F_{n}\left(\zeta^{\prime}\right)-\left[f\left(\zeta^{\prime}, \zeta, \delta\right)\right]^{n} .
\end{aligned}
$$

Thus, from (3.12), we obtain

$$
\left|1-\frac{F_{n}\left(\zeta^{\prime}\right)}{\left[f\left(\zeta^{\prime}, \zeta, \delta\right)\right]^{n}}\right| \leqq \frac{1}{2 \pi} \int_{\sigma_{\rho^{\prime}}}\left|\frac{w}{f\left(\zeta^{\prime}, \zeta, \delta\right)}\right|^{n}\left|\frac{g^{\prime}(w) d w}{g(w)-\zeta}\right| .
$$

Now $\left|g(w)-\zeta^{\prime}\right|=\left|g(w)-\zeta+\zeta-\zeta^{\prime}\right| \geqq|g(w)-\zeta|-\left|\zeta-\zeta^{\prime}\right| \geqq 2 \delta-\delta=\delta$ for $w$ on $\sigma_{\rho^{\prime}}$, and $\left|\zeta^{\prime}-\zeta\right|<\delta$. Also $f\left(\zeta^{\prime}, \zeta, \delta\right) \geqq \rho^{\prime}+\gamma$ for some $\gamma>0$, since if $f\left(\zeta^{\prime}, \zeta, \delta\right)$ $=\rho^{\prime}$, then $g(w)=\zeta^{\prime}$ for $w$ on $\sigma_{\rho^{\prime}}$, in contradiction to the inequality $\left|g(w)-\zeta^{\prime}\right|$ $\geqq \delta$. Thus for $n>n_{0}$,

$$
\left|1-\frac{F_{n}\left(\zeta^{\prime}\right)}{\left[f\left(\zeta^{\prime}, \zeta, \delta\right)\right]^{n}}\right| \leqq\left|\frac{\rho^{\prime}}{\rho^{\prime}+\gamma}\right|^{n}\left|\frac{Q \rho^{\prime}}{\delta}\right|,
$$

where $Q=\max \left|g^{\prime}(w)\right|$ for $w$ on $\sigma_{\rho^{\prime}}$. The estimates used are independent of $\zeta^{\prime}$ for $|\zeta-\zeta|<\delta$, hence for $\left|\zeta^{\prime}-\zeta\right|<\gamma$ and $n>n_{0}$,

$$
\left|1-\frac{F_{n}\left(\zeta^{\prime}\right)}{\left[f\left(\zeta^{\prime}, \zeta, \delta\right)\right]^{n}}\right|<1
$$

Thus, $\Delta$ does not lie in the set $|z-\zeta| \leqq \delta$.

This completes the proof that $C_{1}$ is an open set containing $z=\infty$, and that $\Delta$ lies in the complement of $C_{1}$. The second part of Theorem 1 states that $\Delta$ contains all the boundary points of $C_{1}$. The proof of this depends on the classification of the boundary points of $C_{1}$ and some properties of these classes. These are given in $\$ 4$ and the proof of Theorem 1 is completed in $\$ 5$.

4. The definition of $p$-points and their properties. Part (b) of Theorem 1 requires a classification of the boundary points of $C_{1}$, and some properties of these classes. This material is contained in this section, and the proof of Theorem 2 is completed in the next section.

Definition 4.1. Let $z=g(w)$ be as defined in Definition 3.2 with domain of definition $\Sigma_{\rho}$. Let $\zeta$ be a fixed complex number. If $g(w)-\zeta=0$ has a solution $\omega$ such that $g^{1}(\omega)=\cdots=g^{k-1}(\omega)=0, g^{k}(\omega) \neq 0$, then $\omega$ is a $\zeta$-point of multiplicity $k$. If $k=1$, then $\omega$ is a simple $\zeta$-point. Among the $\zeta$-points of $g(w)$, a finite number, $\omega_{1}, \cdots, \omega_{n}$ will have greatest modulus. Say $\left|\omega_{1}\right|=\cdots=\left|\omega_{n}\right|=\rho^{\prime}$; if 
$p$ is the total multiplicity of the $\zeta$-points, then $\zeta$ is a p-point. If each $\omega_{i}$ is a simple $\zeta$-point, then $\zeta$ is a simple p-point. Otherwise, it is called a multiple p-point.

Lemma 4.1 (Property 1 and 2). Let $\zeta$ be a p-point of $g(w)$, then there is a $\delta>0$, such that if $\left|\zeta^{\prime}-\zeta\right|<\delta, \zeta^{\prime}$ is a q-point, $q \leqq p$. Furthermore it is impossible that each $\zeta^{\prime}$ satisfying $\left|\zeta^{\prime}-\zeta\right|<\delta$ is a p-point.

Proof. Let $\omega_{1}, \cdots, \omega_{n}$ be the solutions of greatest modulus of $g(w)-\zeta=0$, where $\left|\omega_{1}\right|=\cdots=\left|\omega_{n}\right|=\rho^{\prime}$. Since all other solutions have smaller modulus, $\rho^{\prime \prime}$ can be chosen so that $\rho<\rho^{\prime \prime}<\rho^{\prime}$ and no other $\zeta$-points besides $\omega_{1}, \cdots, \omega_{n}$ lie in $\boldsymbol{\Sigma}_{\rho^{\prime \prime}}$. Let $\min |g(w)-\zeta|=2 \delta$ for $w$ on $\sigma_{\rho^{\prime \prime}}$. Then, if $\left|\zeta^{\prime}-\zeta\right|<\delta, g(w)-\zeta^{\prime}$ $=0$ has solutions of total multiplicity $p$ in $\Sigma_{\rho^{\prime \prime}}$. Since these need not be of the same modulus, $\zeta^{\prime}$ is a $q$-point, $q \leqq p$. We refer to this fact as property 1 . The fact that $q=p$ for each $\zeta^{\prime}$ in any $\delta$ neighborhood of $\zeta$ is impossible is called property 2 , and is next proved.

We first note that only a finite number of $\zeta^{\prime}$ satisfying $\left|\zeta^{\prime}-\zeta\right|<\delta$ can be multiple $p$-points. To each multiple $p$-point, $\zeta^{\prime}$, there is a $\zeta^{\prime}$-point in $\Sigma_{\rho^{\prime \prime}}$, say $\omega^{\prime}$, such that $g^{\prime}\left(\omega^{\prime}\right)=0$. Now $g^{\prime}(w)=0$ has only finitely many solutions in $\Sigma_{\rho^{\prime \prime}}$, and is single valued, so our conclusion follows. Thus there is a value $\zeta_{1}$, and $\delta_{1}>0$ satisfying $\left|\zeta_{1}-\zeta\right|<\delta, \delta_{1}<\delta-\left|\zeta_{1}-\zeta\right|$, such that if $\left|\zeta^{\prime}-\zeta_{1}\right|<\delta_{1}$ is a simple $p$-point. The equation $g(w)=\zeta_{1}$ has $p$ solutions $\omega_{1}, \cdots, \omega_{p}$ of greatest modulus, and such that $g^{\prime}\left(\omega_{i}\right) \neq 0$. Thus [2], inverse functions $w=f_{i}\left(z, \zeta_{1}, \delta_{1}\right)$ exist, each holomorphic and univalent for $\left|z-\zeta_{1}\right|<\delta_{1}$ and satisfying the condition $\omega_{i}=f_{i}\left(\zeta_{1}, \zeta_{1}, \delta_{1}\right),(i=1, \cdots, p)$. By hypothesis, each point $z,\left|z-\zeta_{1}\right|<\delta_{1}$ is a $p$-point, hence

$$
\left|\frac{f_{1}\left(z, \zeta_{1}, \delta_{1}\right)}{f_{2}\left(z, \zeta_{1}, \delta_{1}\right)}\right|=1
$$

for $\left|z-\zeta_{1}\right|<\delta$. Therefore

$$
f_{1}\left(z, \zeta_{1}, \delta_{1}\right)=e^{i \alpha} f_{2}\left(z, \zeta_{1}, \delta_{1}\right)
$$

for $\left|z-\zeta_{1}\right|<\delta_{1}$ and $\alpha$ a constant. Now

$$
g\left(f_{1}\left(z, \zeta_{1}, \delta_{1}\right)\right)=g\left(f_{2}\left(z, \zeta_{1}, \delta_{1}\right)\right)
$$

for $\left|z-\zeta_{1}\right|<\delta_{1}$, and therefore

$$
g(w)=g\left(e^{i \alpha} w\right)
$$

for $w$ in an open set. Hence the functional relationship (4.4) must hold in $\Sigma_{\rho}$. However, $z=g(w)$ is univalent in a neighborhood of $w=\infty$, which leads to a contradiction.

Lemma 4.2 (Property 3). Not every point in the neighborhood of a p-point is a 1-point. 
Proof. Case 1. Suppose that $\zeta$ is a simple $p$-point, and the $\zeta$-points of $g(w)$ of greatest modulus are $\omega_{1}, \cdots, \omega_{p}$, each of modulus $\rho^{\prime}$. Choose $\rho<\rho^{\prime \prime}$ $\left\langle\rho^{\prime}\right.$ so that $\bar{\Sigma}_{\rho^{\prime \prime}}$ contains no other $\zeta$-points, and let $c_{i}(i=1, \cdots, p)$ be circles with center at $\omega_{i}$ and lying in $\Sigma_{\rho^{\prime \prime}}$. Let $\min |g(w)-\zeta|=2 \delta$ for $w$ on $\sigma_{\rho^{\prime \prime}}$, $c_{1}, \cdots, c_{p}$. Then if $\left|\zeta-\zeta^{\prime}\right|<\delta$, there is one solution of $g(w)-\zeta^{\prime}=0$ in each circle $c_{i}$. These values are given by holomorphic functions $w=f_{i}(z, \zeta, \delta)$, $(i=1, \cdots, p)$ for $z=\zeta^{\prime}$. These functions are holomorphic for $|z-\zeta|<\delta$, and exist since the conditions for the analytic function $z=g(w)$ to have an inverse, are satisfied. By hypothesis, $\zeta^{\prime}$ is a 1-point, hence one of the functions, say $f_{j}(z, \zeta, \delta)$ yields a point of largest modulus for $z=\zeta^{\prime}$, and we say $\zeta^{\prime}$ is associated with $f_{j}(z, \zeta, \delta)$. In fact, a sufficiently small circle $\sigma^{\prime}$ about $\zeta^{\prime}$ will have an image by $f_{j}(z, \zeta, \delta)$, each point of which has greater modulus than points of the images of $\sigma^{\prime}$ by $f_{i}(z, \zeta, \delta), i \neq j$. Thus the 1 -points in $|z-\zeta|<\delta$ associated with $f_{j}(z, \zeta, \delta)$ form an open set. But then all the points of $|z-\zeta|$ $<\delta$ must be associated with $f_{j}(z, \zeta, \delta)$, for otherwise the open set $|z-\zeta|<\delta$ would be the union of two or more disjoint open sets. From this it follows that

$$
\left|\frac{f_{j}(z, \zeta, \delta)}{f_{i}(z, \zeta, \delta)}\right| \geqq 1, \quad \text { for } i \neq j,|z-\zeta|<\delta,
$$

equality being attained only for $z=\zeta$. This contradicts the maximum modulus theorem, hence property 3 is established when $\zeta$ is a simple $p$-point.

CASE 2. If $\zeta$ is a multiple $p$-point, $g(w)-\zeta=0$ has $k$ solutions of greatest modulus, $\omega_{1}, \cdots, \omega_{k},\left|\omega_{i}\right|=\rho^{\prime}$, of multiplicities $\alpha_{1}, \cdots, \alpha_{k}$ respectively, $\sum \alpha_{i}=p$. As in Case $1, \rho^{\prime \prime}$ is chosen to satisfy $\rho<\rho^{\prime \prime}<\rho^{\prime}$, circles $c_{1}, \cdots, c_{k}$ are chosen about the points $\omega_{1}, \cdots, \omega_{k}$ and a number $\delta$ is found such that if $\left|\zeta^{\prime}-\zeta\right|<\delta, g(w)-\zeta^{\prime}=0$ has solutions of multiplicity $\alpha_{i}$ in $c_{i}(i=1, \cdots, k)$ and no other solutions in $\Sigma_{\rho^{\prime \prime}}$. The inverse function theorem for analytic functions states that the values of $\omega_{j}$ in $c_{i}$ are given by a function $f_{i}\left((z-\zeta)^{1 / \alpha_{i}}\right)$ which is holomorphic in its argument for $|z-\zeta|<\delta$, by using the $\alpha_{i}$ values of the argument. We replace these $k$ multiple valued functions by $p$ single valued holomorphic functions. Replace $f_{i}\left((z-\zeta)^{1 / \alpha_{i}}\right)$ by the $\alpha_{i}$ functions,

$$
\begin{aligned}
& f_{i}^{1}\left(t^{\left(\left(\alpha_{1} \cdots \alpha_{k}\right) / \alpha_{i}\right)}\right), \\
& f_{i}^{2}\left(\rho_{\alpha_{i}} t^{\left(\left(\alpha_{1} \cdots \alpha_{k}\right) / \alpha_{i}\right)}\right) \text {, } \\
& f_{i}\left(\rho_{\alpha_{i}}^{\alpha_{i}-1} t^{\left(\left(\alpha_{1} \cdots \alpha_{k}\right) / \alpha_{i}\right)}\right) \text {, } \\
& (i=1, \cdots, k),
\end{aligned}
$$

where $\rho_{\alpha_{i}}$ is a primitive $\alpha_{i}$ th root of unity. Thus we obtain $p$ functions in all, each single valued in $t$. For a value $t^{\prime},\left|t^{\prime}\right|<\delta^{1 /\left(\alpha_{1} \cdots \alpha_{k}\right)}$, the equation

$$
z-\zeta=\left(t^{\prime}\right)^{\left(\alpha_{1} \cdots \alpha_{k}\right)}
$$


has a unique solution, $\zeta^{\prime}$, satisfying

$$
\left|\zeta^{\prime}-\zeta\right|<\delta
$$

Also

$$
\left(t^{\prime}\right)^{\left(\alpha_{1} \cdots \alpha_{k}\right) / \alpha_{i}}=\left(\zeta^{\prime}-\zeta\right)^{1 / \alpha_{i}} .
$$

Hence the functions (4.6) corresponding to $i$ yield the $\alpha_{i}$ values of $\omega_{j}$ in $c_{i}$. Since $\zeta^{\prime}$ is a 1-point, one of the $p$ functions yields an image of largest modulus, and $\zeta^{\prime}$ is said to be associated with this function. As in the first case, the 1points in $\left|\zeta^{\prime}-\zeta\right|<\delta$ associated with a given function form an open set, and hence all must be associated with the same function. But this, in the same manner, leads to a contradiction of the maximum modulus theorem.

5. Proof of Theorem 1 concluded. We start with the following remarks. If $\zeta$ is a $p$-point, then there are $k \zeta$-points of greatest modulus, $\omega_{1}, \cdots, \omega_{k}$, $(k \leqq p)$, say of modulus $\rho^{\prime}$. Let $F_{n}(z)$ be the Faber polynomial of degree $n$ associated with $f(z)$, then

$$
\lim \sup \left|F_{n}(\zeta)\right|^{1 / n}=\rho^{\prime} .
$$

If $\zeta$ is a 1-point and $\omega$ is the $\zeta$-point of $g(w)$ of greatest modulus, say $|\omega|=\rho^{\prime}$, and $f(z, \zeta, \delta)$ is the inverse of $g(w)$ in the set $|z-\zeta|<\delta$ which satisfies $\omega=f(\zeta, \zeta, \delta)$, then

$$
\lim \left(F_{n}(\zeta)\right)^{1 / n}=f(\zeta, \zeta, \delta),
$$

where the root is chosen so that the expansion begins $\zeta+\cdots$. The weaker form of this is sometimes used,

$$
\lim \left|F_{n}(\zeta)\right|^{1 / n}=\rho^{\prime}
$$

Finally, if $\zeta$ is an 0 -point

$$
\lim \sup \left|F_{n}(\zeta)\right|^{1 / n}=\rho,
$$

where $\Sigma_{\rho}$ is the domain of definition of $g(w)$. These limit relationships are obtained by applying the Cauchy-Hadamard formula for the radius of convergence of a power series to the generating function of the Faber polynomial. Consíder (1.9)

$$
\frac{g^{\prime}(w)}{g(w)-\zeta}=\sum_{0}^{\infty} \frac{F_{n}(\zeta)}{w^{n+1}}
$$

for the case $\zeta$ is a $p$-point, $p>1$. The left side has poles of total multiplicity $p$ on $\sigma_{\rho^{\prime}}$, and no other singularities in $\Sigma_{\rho^{\prime}}$. The formula for the radius of convergence yields (5.1). If $\zeta$ is a 1-point, (5.3) is obtained since the left side of (5.5) has a simple pole on $\sigma_{\rho^{\prime}}$ and no singularities in $\Sigma_{\rho^{\prime}}$. In this case, it is easily shown that (5.2) also holds. Finally if $\zeta$ is an 0-point, (5.3) is obtained, 
since $g(w)$ has a singularity on $\sigma_{\rho}$, whereas in $\Sigma_{\rho}$ the left side of (5.5) has no poles.

The notation $\sigma\left(z_{1}, r\right)$ denotes the closed circle of radius $r$ and center $z_{1}$. Let $\sigma\left(z_{1}, r\right)$ be in the complement of $\Delta$, in which case it will be bounded from $\Delta$. The functions $\left(F_{n}(z)\right)^{1 / n}$, for sufficiently large $n$, have all their branches regular in $\sigma(z, r)$ for otherwise $\sigma\left(z_{1}, r\right)$ would contain a point of $\Delta$. For each $n$, choose one branch, then it will be shown that the sequence of functions forms a normal family in $\sigma\left(z_{1}, r\right)$. In the proof of Part (a) of Theorem 1, it was shown that $\Delta$ lies in the complement of $C_{1}$, and that $C_{1}$ contains the domain $|z| \geqq P, P>0$. Hence for large $n,\left|z_{n i}\right| \leqq P+\delta,(\delta>0)$. But $\max \left|F_{n}(z)\right|^{1 / n}$ $\leqq \max \left|z-z_{n i}\right| \leqq \max |z|+\left|z_{n i}\right|$. For $z$ in $\sigma\left(z_{1}, r\right)$ this is less than $\left|z_{1}\right|+r$ $+P+\delta$. Hence $\left\{\left(F_{n}(z)\right)^{1 / n}\right\}$ forms a normal family in $\sigma\left(z_{1}, r\right)$, and a sequence can be extracted which converges uniformly to a holomorphic function in any interior subset of $\sigma\left(z_{1}, r\right)$.

To complete the proof of Part (b), it is sufficient to consider the following cases. Let $b$ be a boundary point of $C_{1}$.

CASE 1. The point $b$ is a limit point of interior points of $C_{0}$.

CASE 2. Case 1 does not hold, and $b$ is an 0 -point.

CASE 3. Case 1 does not hold, and $b$ is a $p$-point, $p \neq 0$.

Proof of Case 1. Under the hypothesis of this case, it will be shown that $b$ belongs to $\Delta$. Suppose $b$ is not in $\Delta$. Since $\Delta$ is a closed set, there is an $r$ such that $\sigma(b, r)$ does not intersect $\Delta$. By hypothesis, $\sigma(b, r)$ contains an interior point of $C_{0}$, say $z_{0}$. Hence $\sigma(b, r)$ contains $\sigma\left(z_{0}, \gamma\right)$ where $\gamma, \gamma>0$, is chosen so that $\sigma\left(z_{0}, \gamma\right)$ consists of 0 -points. Since by (5.3), lim sup $\left|F_{n}\left(z_{0}\right)\right|^{1 / n}=\rho$, there

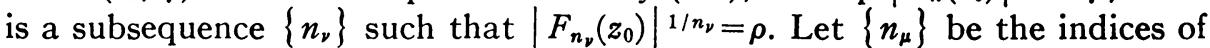
a subsequence of $\left(F_{n_{\nu}}(z)\right)^{1 / n_{\nu}}$ which converges to $F(z)$ in a subset of $\sigma(b, \delta)$ containing $\sigma\left(z_{0}, \gamma\right)$ and a 1-point $\zeta$. Now $\left|F\left(z_{0}\right)\right|=\rho$, and $|F(z)| \leqq \rho$ in $\sigma\left(z_{0}, \gamma\right)$ since points in this circle are 0 -points. Hence $|F(z)|=\rho$ in $\sigma\left(z_{0}, \gamma\right)$ and therefore $F(z)=e^{i \theta_{0} \rho}$ where $\theta_{0}$ is a real constant. Therefore $F(\zeta)=e^{i \theta_{0}}$, but since $\zeta$ is a 1-point, $\lim \left|F_{n_{\mu}}(\zeta)\right|^{1 / n_{\mu}=\rho_{1}}$, where $\rho_{1}>\rho,(5.3)$. This contradiction shows that $b$ belongs to $\Delta$.

CASE 2. Let $b$ be a boundary point of $C_{1}$. From the hypothesis of this case, it follows that there is an $r$ such that $\sigma(b, r)$ contains no point of $\Delta$ and no interior points of $C_{0}$. Hence every point of $\sigma(b, r)$ is a limit of $k$-points $(k=1,2, \cdots)$. By property 1 and 2 of $p$-points, a $k$-point is a limit of $j$ points, $j<k$, hence a limit of 1 -points. In other words, 1-points are dense in $\sigma(b, r)$. By hypothesis $b$ is an 0 -point, thus $\left\{n_{\nu}\right\}$ can be chosen so that $\lim _{\nu \rightarrow \infty}\left|F_{n_{\nu}}(b)\right| 1 / n_{\nu}=\rho$. A further subsequence $\left\{n_{\mu}\right\}$ of $\left\{\eta_{\nu}\right\}$ exists such that in the subset $\sigma(b, \delta / 2), \lim _{\mu \rightarrow \infty}\left(F_{n_{\mu}}(z)\right)^{1 / n_{\mu}}=F(z)$, a holomorphic function. Since 1-points are dense in $\sigma(b, \delta / 2),|F(z)| \geqq \rho$ at a dense set of points, strict inequality being attained at any 1 -point and equality being attained at $b=0$. Since $F(z)$ is continuous, $|F(z)| \geqq \rho$ at each point in $\sigma(b, \delta / 2)$, equality 
being attained for $z=b$. Therefore $F(z)$ is a constant, which involves a contradiction.

CASE 3. In this case $b$ is a boundary point of $C_{1}$, a $p$-point, $(p \geqq 2)$, and by hypothesis not a point of $\Delta$. A number $r, r>0$, can be chosen so that $\sigma(b, r)$ lies in the complement of $\Delta$. By property 3 of $p$ points, not all points in $\sigma(b, \delta)$ are 1-points, hence $b$ is a limit of 2-points. An argument is giver in the proof of Lemma 4.3 showing that only finitely many are multiple points hence $b$ is a limit of simple two points. Let $\zeta$ be one in $\sigma(b, \delta)$. There are two inverse functions, $f_{1}(z, \zeta, \gamma), f_{2}(z, \zeta, \gamma)$, holomorphic for $|z-\zeta| \leqq \gamma$, where $\sigma(\zeta, \gamma)$ is contained in $\sigma(b, \delta)$. These satisfy the conditions $\omega_{1}=f_{1}(\zeta, \zeta, \gamma)$, $\omega_{2}=f_{2}(\zeta, \zeta, \gamma)$, where $\omega_{1}, \omega_{2}$ are the $\zeta$-points of greatest modulus. Since

$$
\left|\frac{f_{1}(z, \zeta, \gamma)}{f_{2}(z, \zeta, \gamma)}\right|=1
$$

for $z=\zeta$, there are two sequences of points $\left\{z_{i}^{\prime}\right\}$ and $\left\{z_{i}^{\prime \prime}\right\}$, both having limit $\zeta$, such that

$$
\left|\frac{f_{1}\left(z_{i}^{\prime}, \zeta, \gamma\right)}{f_{2}\left(z_{i}^{\prime}, \zeta, \gamma\right)}\right|>1, \quad\left|\frac{f_{1}\left(z_{i}^{\prime \prime}, \zeta, \gamma\right)}{f_{2}\left(z_{i}^{\prime \prime}, \zeta, \gamma\right)}\right|<1
$$

Now $\left(F_{n}(z)\right)^{1 / n}$ forms a normal family in $\sigma(b, r)$, hence a subsequence $\left\{n_{\nu}\right\}$ exists such that $\lim \left(F_{n_{\nu}}(z)\right)^{1 / n_{\nu}}=F(z)$, for $z$ in $\sigma(b, \gamma / 2)$, where $F(z)$ is a holomorphic function. Now, by (5.2), and (5.4),

$$
\begin{aligned}
& F\left(z_{i}^{\prime}\right)=f_{1}\left(z_{i}^{\prime}, \zeta, \gamma\right), \\
& F\left(z_{i}^{\prime \prime}\right)=f_{2}\left(z_{i}^{\prime \prime}, \zeta, \gamma\right) .
\end{aligned}
$$

Hence $\lim _{z \rightarrow b} F(z)=\omega_{1}$ for one sequence and $\lim _{z \rightarrow b} F(z)=\omega_{2}$ for the other sequence. This contradiction shows that $b$ belongs to $\Delta$ in Case 3 , and this concludes the proof of Theorem 1.

\section{BIBLIOGRA PHY}

1. G. Faber, Über Polynomische Entwickelungen, Math. Ann. vol. 57 (1903) pp. 389-408.

2. A. Hurwitz and R. Courant, Funktionentheorie, Interscience Publishers, Inc., 1929.

3. R. Jentzsch, Untersuchungen zur Theorie der Folgen analytischer Funktionen, Acta Math. vol. 41 (1917) pp. 219-251.

4. G. Szegö, Über die nullstellen von Polynomen, die in einem Kreise gleichmässig konvergieren, Sitzungsberichte der Berliner Mathematische Gesellschaft vol. 21 (1922) pp. 59-64.

University of Michigan, AnN Arbor, Michigan 